\title{
MONOPOLES, DUALITY, TRIALITY
}

\author{
X。 Artru $\left.{ }^{*}\right)$
}

CERN - Geneva

\section{A $B$ S $T$ T $R$ A $\quad C \quad T$}

The existence of magnetic monopoles could provide an explanation not only of the quantization of electric charge, but also of the zero triality and of the string model with $Y$ shaped baryons. First we review the classical magnetic monopole theory with strings and the Dirac least action principle. Two possible interaction terms, the standard one $j \cdot A$ and a path dependent interaction à la Mandelstam give the same classical equations of motion, and the strings are not observable. At the quantum level, this is not true anymore. Assuming a linear combination of the two interaction terms, we determine the condition under which a string attached to the dyon $\left(e_{1}, g_{1}\right)$ is not observable by a dyon (e $\left.e_{2}, g_{2}\right)$, using a "sum over histories" approach. We get $(1-\mathrm{x}) \mathrm{e}_{2} \mathrm{~g}_{1}-\mathrm{xe}_{1} \mathrm{~g}_{2}=2 \pi \times$ integer. The Dirac and Schwinger-Zwanziger quantization rules are special cases, with $\mathrm{x}=0$ and $\frac{1}{2}$, respectively. Then, assuming the existence of magnetic charges, we look for all possible models where : 1) the string attached to an electron is not observable, 2) the string attached to a quark is observable, 3) the electric charge has to be quantized in units e/3. We find a denumerable set of models where the magnetic charges may also be connected with physical strings.

*) Address after 1 October 1976 : LPTHE, Université de Paris XI, Bâtiment 210, 91405 Orsay. 
Magnetic monopoles 1),2), if they exist, would provide an explanation for the quantization of electric charge. They could also serve to confine fractionally charged quarks into integrally charged clusters, as was first proposed by schiff ${ }^{3)}$. On the other hand, the most simple mathematical object which can confine the quarks is an infinitely thin string and the string model of the hadron 4) has more than one phenomenological success : duality, linear trajectories... It can also describe classically the most important features of high energy multiproduction; leading particle effect, limiting fragmentation, small transverse momenta, pionization... 5).

It is possible to reconcile both ideas for the confinement, saying that magnetic monopoles lead to physical strings which confine the quarks. We have exposed the basic idea in a previous paper ${ }^{6}$ ). Here we develop this hypothesis in more detail.

In Section 2, we review the Dirac formulation for the classical theory of magnetic monopoles. A modification of Dirac's action is proposed, using Mandelstam's path dependent quantities. The two results are compared. The generalization to a system of dyons connected with physical and unphysical strings is made in section 3. In Section 4, we discuss the quantum mechanical effect of two strings passing through each other, and set down the condition for a string to be spurious (a generalization of Dirac or Schwinger conditions). Mandelstam's and conventional interactions differ by a "contact term", which introduces a parameter $\mathrm{x}$ in the theory. In Section 5, we try to see what the magnetic charges and $\mathrm{x}$ must be, in order to explain that : 1) electrons, protons, etc., are free ; 2) all charges are multiples of $\mathrm{e} / 3$; 3) quarks are not free. A denumerable set of solutions is found, and we display the most simple ones. In Section 6, we discuss these results, from the theoretical and experimental point of views. 
$-2-$

2. - CLASSICAL THEORY OF MAGNETIC CHARGES : DIRAC'S LEAST ACTION PRINCIPLE

In the presence of both electric and magnetic charges, the Maxwell equations become

$$
\begin{aligned}
& \operatorname{div} \vec{E}=j^{0} \\
& \text { (2.1a) } \\
& -\frac{\partial \vec{E}}{\partial t}+\operatorname{curl} \vec{H}=\vec{j} \\
& (2.1 b) \\
& \operatorname{div} \vec{H}=k^{0} \\
& (2 \cdot 2 a) \\
& -\frac{\partial \vec{H}}{\partial t}-\operatorname{curl} \vec{E}=\vec{k} \\
& j^{0}(\vec{x}, t)=e \delta^{3}[\vec{x}-\vec{x}(t)] \\
& \vec{j}(\vec{x}, t)=j(\vec{x}, t) \vec{v}
\end{aligned}
$$

where $\vec{v}$ is the velocity. Similarly, for a magnetic charge $(e \rightarrow g, j \rightarrow k)$. Let us write these equations in covariant form. Defining

$$
\begin{aligned}
& F_{k \nu}=\left(\begin{array}{cccc}
0 & E_{x} & E_{y} & E_{z} \\
-E_{x} & 0 & -H_{z} & H_{y} \\
-E_{y} & H_{z} & 0 & -H_{x} \\
-E_{z} & -H_{y} & H_{x} & 0
\end{array}\right) \\
& \tilde{F}=-\frac{1}{2} \varepsilon_{\mu \nu \rho r} \\
& F^{p r}=\left(\begin{array}{cccc}
0 & H_{x} & H_{y} & H_{z} \\
-H_{x} & 0 & E_{z} & -E_{y} \\
-H_{y} & -E_{z} & 0 & E_{x} \\
-H_{z} & E_{y} & -E_{x} & 0
\end{array}\right)
\end{aligned}
$$


$-3-$

they become

$$
\begin{aligned}
& \partial_{\mu} F^{\mu \nu}=j^{\nu} \\
& \partial_{\mu} \tilde{F}^{\mu \nu}=k^{\nu}
\end{aligned}
$$$$
j^{\mu}(x)=e \int_{\text {trajectory }} d x^{\mu} \delta^{4}(x-X)
$$$$
k^{\mu}(y)=g \int_{\text {raj. }} d Y^{\mu} \delta^{4}(y-Y)
$$

Equations (2.2) and (2.6) give one of the two possible definitions of a magnetic charge. It is something surrounded (at rest) by a coulomb-like magnetic field $\overrightarrow{\mathrm{H}}=\mathrm{gr} / 4 \pi r^{3}$. The other definition involves the electromagnetic force acting on it. For the electric case we have

$$
\begin{aligned}
& \vec{F}=\frac{d \vec{P}}{d t}=e(\vec{E}+\vec{v} \times \vec{H}) \\
& d P_{0}=e \vec{E} \cdot d \vec{x} \\
& d P_{\mu}=e F_{\mu \nu} d x^{\nu}
\end{aligned}
$$

For a magnetic charge, we require :

$$
d P_{\mu}=g \tilde{F}_{\mu \nu} d x^{\nu}
$$$$
(2.8 a)
$$

which means

$$
\vec{F}=g(\vec{H}-\vec{v} \times \vec{E})
$$


$-4-$

The two definitions, (2.2) and (2.8), of magnetic charges are compatible : one can verify that energy momentum is conserved by the interaction; taking for the energy momentum tensor of the field

$$
T_{\alpha}^{\beta}\left(f_{\text {field }}\right)=\frac{1}{4} g_{\alpha}^{\beta} F^{\mu \nu} F_{\mu \nu}-F_{\alpha \tau} F^{\beta \tau}
$$

(in this expression $F$ can be replaced by $\widetilde{F}$ ), we have

$$
\begin{aligned}
\partial_{\beta} T_{\alpha}^{\beta}\left(F_{\text {ied }}\right) & =-F_{\alpha \tau} \partial_{\beta} F^{\beta \tau}-\tilde{F}_{\alpha \sigma} \partial_{\beta} \tilde{F}^{\beta r} \\
& =-F_{\alpha \tau} j^{\tau}-\tilde{F}_{\alpha \sigma} k^{\sigma}
\end{aligned}
$$

while (2.7) and (2.8) are equivalent to

$$
\partial_{\beta} T_{\alpha}^{\beta} \text { (electric charge) }=F_{\alpha \tau} j^{\tau}
$$

$$
\left.\partial_{\beta} T_{\alpha}^{\beta} \text { (magnetic charge) }\right)=\tilde{F}_{\alpha \sigma} k^{\sigma}
$$

There is a general agreement on the equations of motion (2.1), (2.2), (2.7) and (2.8). They are invariant under the duality transformation

$$
\begin{aligned}
& \left.\begin{array}{l}
E \rightarrow H \\
H \rightarrow-E
\end{array}\right\} \text { or }\left\{\begin{array}{l}
F \rightarrow \tilde{F} \\
\tilde{F} \rightarrow-F
\end{array}\right. \\
& \left.\begin{array}{l}
e \rightarrow g \\
g \rightarrow-e
\end{array}\right\} \text { or }\left\{\begin{array}{lll}
j & \rightarrow k \\
k & \rightarrow & -j
\end{array}\right.
\end{aligned}
$$
field 


$$
F_{\mu \nu}+\varphi_{\mu \nu}=\partial_{\mu} A_{\nu}-\partial_{\nu} A_{\mu}
$$

$\varphi$ is a delta function on the string (finite flux in zero thickness);

$$
\begin{aligned}
& \vec{H}^{\varphi}=g \delta^{2}\left(\vec{x}_{\perp}\right) \vec{\tau} \\
& \vec{E}^{\varphi}=\vec{v} \times \vec{H}^{\varphi}
\end{aligned}
$$

where $\vec{x}_{\perp}$ is the shortest distance from $x$ to the string, $\vec{\tau}$ the unit tangent vector oriented toward the charge, $\vec{v}$ the local velocity of the string. A is singular on the string and $F$ is regular. This decomposition is visualized in Fig. 1.

By construction [Eq. (2.15a $], \overrightarrow{\mathrm{H}}^{\varphi}$ is to satisfy

$$
\operatorname{div} \vec{H}^{\varphi}=-k^{0}
$$

which yields (2.2a).

Let us define the tensor area, swept by a piece of string in space time, as

$$
\Sigma^{\mu \nu}=\frac{1}{2} \oint x^{\mu} d x^{\nu}-x^{\nu} d x^{\mu}=\oint x^{\mu} d x^{\nu}
$$

(see Fig. 2 for the integration contour). The infinitesimal area swept by an element $\overrightarrow{\mathrm{d} l}$ is

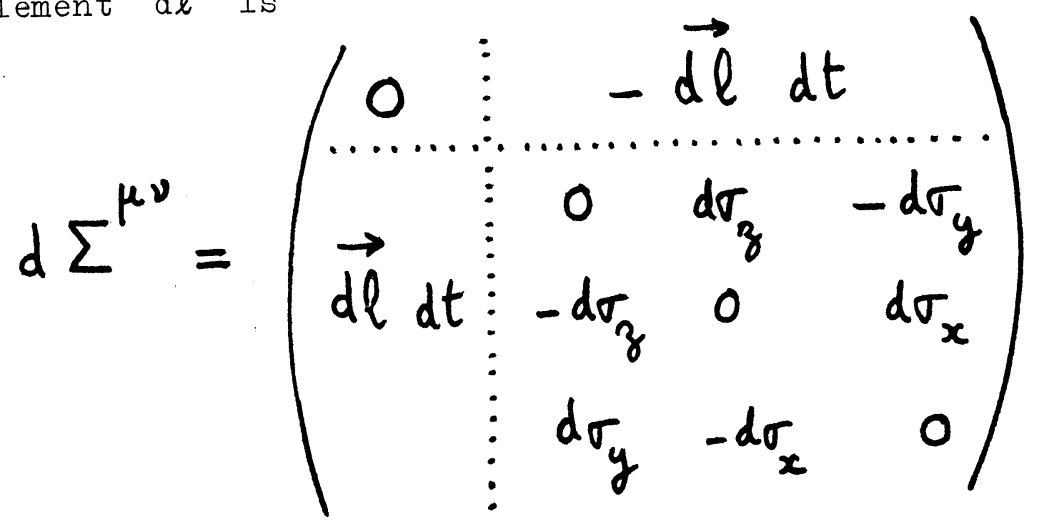


$-6-$

with $d \vec{\sigma}=\overrightarrow{d l} \times \vec{v}$ at. In covariant notation, if we denote the displacement by dy, we have

$$
d \Sigma^{\mu \nu}=d l^{\mu} d y^{\nu}-d l^{\nu} d y^{\mu}
$$

Equations $(2.15 a)$ and $(2.15 b)$ can be written covariantly as

$$
\tilde{\varphi}^{\mu \nu}(x)=-g \int \delta^{4}(x-X) d \Sigma^{\mu \nu}
$$

and $(2.16 a)$ generalizes as

$$
\partial_{\mu} \tilde{\varphi}^{\mu \nu}=-k^{\nu}
$$

Then Dirac proceeds as follows : the action is a sum of three terms :

$$
A \text { (particles) }=-\sum_{\text {particles }} m d s
$$

$$
\begin{aligned}
& A(\text { field })=-\frac{1}{4} \int F^{\mu \nu} F_{\mu \nu} d^{4} x=\frac{1}{2} \int\left(\vec{E}^{2}-\vec{H}^{2}\right) d^{4} x \\
& A(\text { (interaction })=-\int j^{\mu} A_{\mu} d^{4} x=-\sum_{\text {el. charges }} e \int_{\text {tray }} A_{\mu} d_{\mu^{\mu}} \quad \text { (2.20) } \\
& \text { only the electrically charged particles have an explicit inter- }
\end{aligned}
$$
action term with the field. The interaction for the magnetic monopoles is contained in $t$ (field), through the $\varphi_{\mu \nu}$ dependence. Dirac's treatment is not dual-symmetric but this seems unavoidable, since the lagrangian density of the free field, $\frac{1}{2}\left(\overrightarrow{\mathrm{E}}^{2}-\overrightarrow{\mathrm{H}}^{2}\right)$ is antisymmetric, whereas the Lagrangin of the particles is symmetric. On the other hand, the theory can be formulated with the dually opposite convention, ide., interchanging the rôles of electricity and magnetism. Before introducing physical strings, let us show how one gets the correct equations of motion, in the case of fictitious strings only. 
A) - Variation of the electric charge trajectory

A simple calculation gives

$$
\delta A(\text { particle })=\int_{\text {traj. }} \delta X \cdot d P
$$

with $P=m d x / d s$,

$$
\begin{aligned}
\delta A_{(\text {interaction })} & =-e \int_{\text {traj. }}\left(\partial_{\mu} A_{\nu}-\partial_{\nu} A_{\mu}\right) d x^{\nu} \delta X^{\mu} \\
& =-e \int_{\text {traj. }} \delta X^{\mu} F_{\mu \nu} d X^{\nu}
\end{aligned}
$$

(provided $\varphi_{\mu \nu}=0, i . e$. , the trajectory does not pass through a magnetic string).

Adding (2.21) and (2.22) must yield zero : one gets (2.7c).

B) - Variation of the vector potential

We have

$$
\delta\left(F^{\mu \nu} F_{\mu \nu}\right)=2 F^{\mu \nu} \delta F_{\mu \nu}=2 F^{\mu \nu} \delta\left(\partial_{\mu} A_{\nu}-\partial_{\nu} A_{\mu}\right)=4 F^{\mu \nu} \partial_{\mu} \delta_{\nu}
$$

After an integration by part, we get

$$
\begin{aligned}
& \delta A(\text { field })=\int \delta A_{\nu} \partial_{\mu} F^{\mu \nu} d^{4} x \\
& \delta A\left(\text { interaction) }=-\int \delta A_{\nu} j^{\nu} d^{4} x\right.
\end{aligned}
$$

These two variations must cancel each other : we get (2.1c)。 Equation (2.2c) is "built-in", by (2.14).

\section{c) - Variation of the history of a magnetic string}

It includes a variation of the trajectory of the magnetic charge, and we have (2.21) again for the action of the particle. This time, it is the action of the field which varies : 
$-8-$

$$
\begin{gathered}
\delta F=-\delta \varphi\left(A_{\mu} \text { is kept constant }\right), \\
\delta A\left(F_{\text {eld }}\right)=\frac{1}{2} \int F_{\mu \nu} \delta \varphi^{\mu \nu} d^{4} x
\end{gathered}
$$

(We have to neglect terms proportional to $\mathrm{g}^{2}$ )

$$
\begin{aligned}
& =-\frac{1}{2} \int \tilde{F}_{\mu \nu} \delta \tilde{\varphi}^{\mu \nu} d^{4} x \\
& =-\frac{g}{2}\left(\int_{\Sigma_{1}}-\int_{\Sigma_{2}} \tilde{F}_{\mu \nu} d \Sigma^{\mu \nu}\right)
\end{aligned}
$$

where $\Sigma_{1}$ and $\Sigma_{2}$ are the old and new sheets swept by the string [we have used (2.15c $]$. Provided no electric charge passes between the two histories, one can replace $\int_{\Sigma_{2}}-\int_{\Sigma_{1}}$ by $\int_{\delta \Sigma}$, where $\delta \Sigma$ is the two-dimensional surface swept by the $\delta X$ of the charge trajectory (see Fig. 3). This comes from a generalization of Gauss' law ${ }^{*}$ )

$$
\frac{1}{2} \int \tilde{F}_{\mu \nu} d \Sigma^{\mu \nu}=\int d V_{\nu} \partial_{\mu} F^{\mu \nu}=\int d V_{\nu} j^{\nu}
$$

for a closed two-dimensional surface $\Sigma$ enclosing a three-dimensional surface $V$. Therefore

$$
\begin{aligned}
\delta A\left(F_{\text {field }}\right) & =-\frac{g}{2} \int_{\delta \Sigma} \tilde{F}_{\mu \nu} d \Sigma^{\mu \nu} \\
& =-g \int_{t_{\text {raj. }}} \tilde{F}_{\mu \nu} \delta X^{\mu} d X^{\nu}
\end{aligned}
$$

*) Our convention is, for the three-dimensional infinitesimal element swept by the element $d \Sigma^{\nu \mu}$ in a displacement $\mathrm{dz}$,

$$
d V_{\mu}=\frac{1}{2} \varepsilon_{\mu \nu \rho \sigma} d \Sigma^{\nu \rho} d z^{\sigma}=\varepsilon_{\mu \nu \rho \sigma} d x^{\nu} d y^{\rho} d z^{\sigma}
$$


$-9-$

Equation (2.29) is dual symmetric of (2.22). We see that the string variables play no dynamical rôle, at least classically, and when no electric charge passes through a magnetic string (but, classically, this type of event has a zero probability.)

Thus we have got all the equations of motion from Dirac's action principle. They are dual symmetric and, in fact, one could have started from

$$
\tilde{F}_{\mu \nu}=\partial_{\mu} B_{\nu}-\partial_{\nu} B_{\mu}-\tilde{\psi}_{\mu \nu}
$$

instead of (2.14), with "electric strings" instead of "magnetic" ones, etc.

Mandelstam

9) has proposed a path dependent formalism for usual quantum electrodynamics, where the interaction is expressed in terms of the field $F_{\mu \nu}$ instead of the potential $A_{\mu}$. In quite a similar way, at the classical level, we can replace the interaction term (2.20) by

$$
A \text { (interaction) }=-\frac{1}{2} \sum_{\text {elect } \text { charges }} e \int_{\Sigma} F_{\mu \nu} d \Sigma^{\mu \nu}
$$

where the integration is performed on a two-dimensional surface $\Sigma$ swept by a fictitious string coming from infinity to the electric charge. Let us consider, indeed, a finite region $\mathrm{R}$ of spacetime where we vary the brajectory, the fictitious string motion and the potential. Outside $R$, and on its boundary $\partial R$, we take the variations to be zero (this is not really a restriction, since $\mathrm{R}$ can be arbitrarily large). We have (generalization of Stoke's theorem)

$$
\int_{\text {tray. }}-\int_{I} A_{\mu} d x^{\mu}=\frac{1}{2} \int_{\Sigma_{R}}\left(\partial_{\mu} A_{\nu}-\partial_{\nu} A_{\mu}\right) d \Sigma^{\mu \nu}
$$

where $I$ is the intersection of $\Sigma$ with $\partial R$ and $\Sigma_{R}$ the part of $\Sigma$ contained in $R$. If the fictitious (electric) string does not cross a magnetic string, we can replace $\partial_{\mu} A_{\nu}-\partial_{\nu} A_{\mu}$ by $F_{\mu \nu}$ in (2.32). On the left-hand side of (2.32), only the first term varies. This establishes that for the variations, (2.31) and (2.20) are equivalent. Now, if there is a magnetic string on the way, (2.32) has a "crossing term"

$$
C=-\frac{e}{2} \int_{\Sigma} \varphi_{\mu \nu} d \Sigma^{\mu \nu}
$$


in addition to (2.31). This term is a constant and can be evaluated using a particular frame where the magnetic string is at rest at the crossing point (Fig. 4). From (2.4) and (2.17b), we have

$$
\frac{1}{2} \varphi_{\mu \nu} d \Sigma^{\mu \nu}=-\vec{H}^{\varphi} \cdot \overrightarrow{d \sigma}-\vec{E}^{\varphi} \cdot \overrightarrow{d l} d t
$$

$\vec{E}^{\varphi}=0$ in our frame and $\int \vec{H}^{\varphi} \cdot d \vec{\sigma}$ is the flux carried by the magnetic string. Its absolute value is $g$ and its sign depends on the orientation of the triad formed by the tangent vectors $\vec{\tau}_{e}, \vec{\tau}_{g}$ to the strings and their relative velocity $\overrightarrow{\mathrm{v}}_{\mathrm{e} / \mathrm{g}}$ :

$$
C=e g \times \operatorname{sign}\left(\vec{\tau}_{e}, \vec{v}_{e / g}, \vec{\tau}_{g}\right)
$$

Thus we have completed the proof of the equivalence of the two choices of action (2.31) and (2.20). It is to be noted, however, that $C$ is not defined when the electric string meets the magnetic charge, and we expect that the equivalence is valid only at the classical level. Up to now, the strings were only a mathematical tool and played no dynamical rôle. This is still valid at the quantum level if the Dirac quantization condition for the charges is satisfied, as we shall see in section 4. This is satisfactory for the conventional point of view, where one wants the strings to be unobservable. We shall not make such a requirement and allow some strings to be observable.

\section{3. - CLASSICAI THEORY OF PHYSICAL STRINGS WITH ELECTRIC AND MAGNETIC CHARGES AT THEIR END POINTS}

Essentially we keep the Dirac formulation, but take the second form (2.31) for $t$ (interaction) and give to some of the strings (those which we want to be observable) a linear mass density as in the conventional string model for the hadrons. Therefore we add to the action a term 
- $11-$

proportional to the invariant area swept by the string. For generality, we shall consider dyons of charges $\left(e_{i}, g_{i}\right)$ instead of pure electric or pure magnetic charges. The attached strings will be mixtures of "electric" and "magnetic strings". The action has now four pieces :

$$
\begin{aligned}
& A(\text { field })=-\frac{1}{4} \int F_{\mu \nu} F^{\mu \nu} d^{4} x \\
& A(\text { interaction })=-\frac{1}{2} \sum_{i} e_{i} \int F_{\mu \nu} d \Sigma^{\mu \nu} \\
& A(\text { particles })=-\sum_{i} m_{i} \int d s \\
& A(\text { strings })=-\sum_{i} k_{i} \int d \Sigma
\end{aligned}
$$

where

$$
\begin{aligned}
& F^{\mu \nu}=\partial_{\mu} A_{\nu}-\partial_{\nu} A_{\mu}-\varphi^{\mu \nu}, \\
& \tilde{\varphi}^{\mu \nu}=-\sum_{i} g_{i} \int \delta^{4}(x-x) d \Sigma^{\mu \nu} \\
& d \Sigma=\sqrt{-d \Sigma^{\mu \nu} d \Sigma_{\mu \nu} / 2}
\end{aligned}
$$

and $x_{i}$ is the linear mass density or the tension of the string at rest.

The variations of the first three terms have been given in the preceding section. The variation of the string action is a well-known problem : we parametrize the sheet as $X^{\mu}(\sigma, \tau)$. The dot and prime denote differentiation with respect to $T$ and $\sigma$, respectively. Accordingly,

$$
d \Sigma=\sqrt{\left(\dot{X} \cdot x^{\prime}\right)^{2}-(\dot{X} \cdot \dot{x})\left(x^{\prime} \cdot x^{\prime}\right)} d \sigma d \tau
$$

(in shorthand notation $: \sqrt{ } d \sigma d r)$. One finds 
$-12-$

$$
\begin{aligned}
\delta A(\text { string }) & =x \int_{\Sigma} \delta X \cdot\left(\partial_{\tau} \frac{\partial \sqrt{ }}{\partial \dot{x}}+\partial_{\sigma} \frac{\partial \sqrt{ }}{\partial X^{\prime}}\right) d \sigma d \tau \\
& -x \int_{\text {traj. }} \delta X \cdot\left(\frac{\partial \sqrt{r}}{\partial X^{\prime}} d \tau-\frac{\partial \sqrt{ }}{\partial \dot{x}} d \sigma\right)
\end{aligned}
$$

The first integral has no counter part in $\delta \cdot t$ (total), therefore

$$
\partial_{\tau} \frac{\partial \sqrt{ }}{\partial \dot{x}}+\partial_{\sigma} \frac{\partial \sqrt{ }}{\partial x^{\prime}}=0
$$

This is the well-known equation of the string which, with an orthonormal parametrization $\left(\dot{X} \cdot X^{\prime}=0, \dot{X} \cdot \dot{X}=-X^{\prime} \cdot X^{\prime}\right)$, writes

$$
\ddot{x}=x^{\prime \prime}
$$

the second integral can be written as

$$
\delta A(\text { boundary })=-x \int_{\text {traj. }} \delta X \cdot \widetilde{d X}
$$

where $\widetilde{d X}$ is the "dual" vector of $d x$ in the sheet (another kind of dualty !), defined as follows :

1) $d X \cdot \tilde{d X}=0$

2) $d \widetilde{X}_{0} d \widetilde{X}=-d X \cdot d X$

3) $d \widetilde{X}$ is tangent to the sheet and is directed inside it. Its geometrical construction, using the light lines on $\Sigma$, is very simple (see Fig. 5).

We can define a covariant tension by

$$
\begin{aligned}
& T^{\mu}=x \widetilde{d X}^{\mu} / d s \\
& T \cdot T=-x^{2}
\end{aligned}
$$


It represents the force exerted by the string on the particle. This is the only modification for the equation of motion of the particle. Instead of (2.7) or (2.8), we have :

$$
d P_{\mu}(\text { particle } i)=\left(e_{i} F_{\mu \nu}+g_{i} \widetilde{F}_{\mu \nu}\right) d X^{\nu}+x_{i} \widetilde{d X}_{\mu} \text { (3.13) }
$$
The field exerts no force on the strings except at their end points.

At a three-string junction, we have to balance the tensions defined by (3.12) ( $s$ is the proper time of the junction)

$$
T_{\mu}(1)+T_{\mu}(2)+T_{\mu}(3)=0
$$

Chiral transformation

Once we admit dyons, what is called "electric" or "magnetic" is a matter of convention. Indeed, one can make a rotation in the "electric" vs. "magnetic" plane :

$$
\left.\begin{array}{l}
\vec{E}^{\prime}=\vec{E} \cos \theta+\vec{H} \sin \theta, \\
\vec{H}^{\prime}=-\vec{E} \sin \theta+\vec{H} \cos \theta, \\
F^{\prime}=F \cos \theta+\widetilde{F} \sin \theta, \\
e_{i}^{\prime}=e_{i} \cos \theta+g_{i} \sin \theta, \\
g_{i}^{\prime}=-e_{i} \sin \theta+g_{i} \cos \theta,
\end{array}\right\}
$$

etc. The equations of motion with the prime quantities are the same. $\theta=\pi / 2$ corresponds to the dual transformation. By a suitable rotation, one can make one of the dyons a pure electric charge.

This Lagrangian formulation is not chiral-invariant, and we expect that a straightforward quantization will give non-chiral invariant effects. 


\section{4. - QUANTUM OBSERVABILITY OF A STRING}

Here we shall not give a quantum theory of strings, charges and monopoles. We only assume that such a theory exists and may be derived with the "sum over histories" method 10) (which is the analogue of the classical least action principle). The problem is the following : does a string which is not observable at the classical level $(x=0)$ become observable at the quantum level (through a kind of Bohm-Aharonov effect) ? Let us consider therefore a dyon of charge $\left(\mathrm{e}_{2}, \mathrm{~g}_{2}\right)$ passing in the vicinity of a string carcarrying flux $\left(e_{1}, g_{1}\right)$ (Fig. 6$)$, and see if there is an interference between the two paths. According to the sum over histories formalism, the contribution of a path $P$ to the amplitude has the phase factor

\section{i $A(P) / \hbar$ \\ e}

According to what we have seen in the preceding section, the action $f(P)$ is a continuous function of $P$ and does not depend on the string co-ordinates at least when dyon 2 does not pass through string 1 . In this case, we have a discontinuity $D=t(P)-t\left(P^{\prime}\right)$, and this is the quantity that we want to calculate. For if ${ }^{*}$ )

$$
D \equiv 0 \quad(2 \pi)
$$

string 1 can be ignored physically.

The only term which contributes to $D$ is $t$ (interaction) Eq. (3.2). A simple way to get it is the following : we choose a frame where dyon 2 is at rest and string 1 is moving sweeping two possible sheets (Fig. 7)

$$
\begin{aligned}
D & =\int_{\Sigma^{\prime}}-\int_{\Sigma} \frac{e_{1}}{2} F_{k \nu} d \Sigma^{\mu \nu} \\
& =\int_{\Sigma}-\int_{\Sigma^{\prime}} e_{1}(\vec{H} \cdot \overrightarrow{d r}+\vec{E} \cdot \overrightarrow{d l} d t)
\end{aligned}
$$

*) We put $\hbar=1$. 
$-15-$

In the vicinity of 2 , we have

$$
(\vec{E}, \vec{H})=\left(e_{2}, g_{2}\right) \frac{\vec{r}}{4 \pi r^{3}}
$$

The contribution of $\overrightarrow{\mathrm{E}}$ vanishes. Applying Gauss' law for $\overrightarrow{\mathrm{H}}$, we get a first result

$$
D=-e_{1} g_{2}=D_{a}
$$

This result is not chiral invariant and comes from our choice of the interaction term. (2.31)-(3.2). If, instead, we take the standard interaction term, in $j^{\mu} A_{\mu}[E q .(2.20]$, we get the same classical equations of motion but a different D. Let us now consider Fig. 6 :

$$
D=\int_{p^{\prime}}-\int_{P} e_{2} d x^{\mu} A_{\mu}
$$

The calculation is identical to that of the constant C [Ers. (2.32), (2.33), (2.34 . We get the Dirac result :

$$
D=+e_{2} g_{1}=D_{b}
$$

which is not chiral-invariant either. We can also take for the interaction a linear combination of $(2.20)$ and (2.31), which gives

$$
D=(1-x) e_{2} g_{1}-x \quad e_{1} g_{2}
$$

For $x=\frac{1}{2}$ we get the chiral-invariant Schwinger-Zwanziger 11) result :

$$
D=\frac{1}{2}\left(e_{2} g_{1}-e_{1} g_{2}\right)=D_{c}
$$


$-16-$

If, before calculating $D$, we make a chiral rotation (3.15), the new action will be, in terms of the old quantities $e$ and $g$

$$
\begin{aligned}
& 2 D=e_{2} g_{1}-e_{1} g_{2}+(1-2 x)\left[\left(e_{2} g_{1}+e_{1} g_{2}\right) \cos 2 \theta\right. \\
& \left.-\left(e_{1} e_{2}-g_{1} g_{2}\right) \sin 2 \theta\right]
\end{aligned}
$$

This is a more general form for $D$ than (4.4). However, we prefer to restrict ourselves to the form (4.4) for the following reason : let us consider a system of two electrons. Their strings are not observale so

$$
0 \equiv D=e^{2}(x-1 / 2) \sin 2 \theta \quad(2 \pi)
$$

if $D \neq 0,|x|$ has to be very large $(\gtrsim 137 / 2)$. For aesthetical reasons, we assume

$$
(1-2 x) \sin 2 \theta=0
$$

in which case $(4.5)$ reduces to $(4.4)$.

5. - DIFFERENT POSSIBLE MODELS FOR THE PHYSICAL WORID

Generalizing Dirac's method and using a "sum over histories" argument, we have obtained a condition for the string attached to a deon 1 to be not observable by a dion 2 :

$$
D=(1-x) e_{2} g_{1}-x e_{1} g_{2} \equiv 0 \quad(2 \pi), \quad(5.1)
$$

where $x$ is, for the moment, an unknown parameter. Now we shall take into account the following experimental facts (or hypotheses) :

1) the electron is "free" (= not confined); this means

$$
x \in g_{i} \equiv 0 \quad(2 \pi) \text {, for all } g_{i}
$$


- $17-$

2) the quarks have pure electric charges $2 \mathrm{e} / 3,-\mathrm{e} / 3$, and are not free ; we assume that this is due to the existence of at least one (free or not free) dyon which does not satisfy (5.1) and "see" the strings of the quarks ; let $(\epsilon, \gamma)$ be such a dion which has the smallest possible magnetic charge in absolute value :

$$
x e \gamma / 3 \neq \equiv \quad(2 \pi)
$$

3) the electric charge is quantized (this was the original motivation to consider monopoles), in multiple of $\mathrm{e} / 3$; this fact allows the existthence of a free lyon, and we assume that it exists, and has the smallest allowed magnetic charge. Let us call $e^{\prime}$ and $g$ its charges.

$$
\begin{aligned}
& (1-x) \mathrm{eg} / 3=+2 \pi \\
& (1-2 x) e^{\prime} g \equiv 0 \quad(2 \pi)
\end{aligned}
$$

and to any other deon

$(1-x) e_{i} g-x e^{\prime} g_{i} \equiv 0 \quad(2 \pi)$, for all $i$

From (5.2) and $(5.3)$, we have

$$
\begin{aligned}
& x \text { eg } / 2 \pi=m, \text { integer } \neq 0 \\
& x \text { er } / 2 \pi=k, \text { integer } \\
& \text { but not multiple of } 3
\end{aligned}
$$

By charge conjugation, we can choose $\gamma / g>0$. With our definition of $\gamma$,

$$
\left\{\begin{array}{l}
|\gamma| \leqslant|g| / 2 \\
\text { or } \gamma=g,
\end{array}\right.
$$


otherwise we could form a compound dyon from $|\gamma|$ and $-|g|$ with a smaller magnetic charge.

$$
\begin{aligned}
& \text { From (5.4) and }(5.7) \text {, we get } \\
& \qquad x=\frac{m}{3+m}
\end{aligned}
$$

$$
e g=2 \pi(3+m)
$$

and from $(5.7),(5.8)$ and $(5.9)$

$$
\gamma / g=k / m\left\{\begin{array}{l}
\leqslant 1 / 2 \\
\text { or }=1
\end{array}\right.
$$

We see that there exists an infinite number of models, which can be classified by $\mathrm{m}$, and $\mathrm{k}$ (and possible electric charges for the dyons). $m$ gives the value of the free magnetic monopole. If $k=m$, all magnetic monopoles are free. If $k<m$, there exist magnetic analogues of the quarks, with "non-integer magnetic charges" connected by strings. Let us see an example:

$\mathrm{m}=-2, \mathrm{k}=-1$; the smallest free magnetic charge is $\mathrm{g}=\mathrm{e} \times 137 / 2$. It can be shared by two "magnetic quarks" $\gamma=\frac{1}{2} g$, connected by a string. We have two kinds of "magnetic meson"

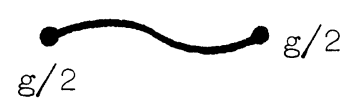

and

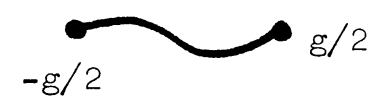

This string is not oriented. From (5.5) and (5.6), the magnetic quark could be a dyon of electric charge $e / 5 \times$ integer.

We give in the following Table some of the most simplest solutions. 


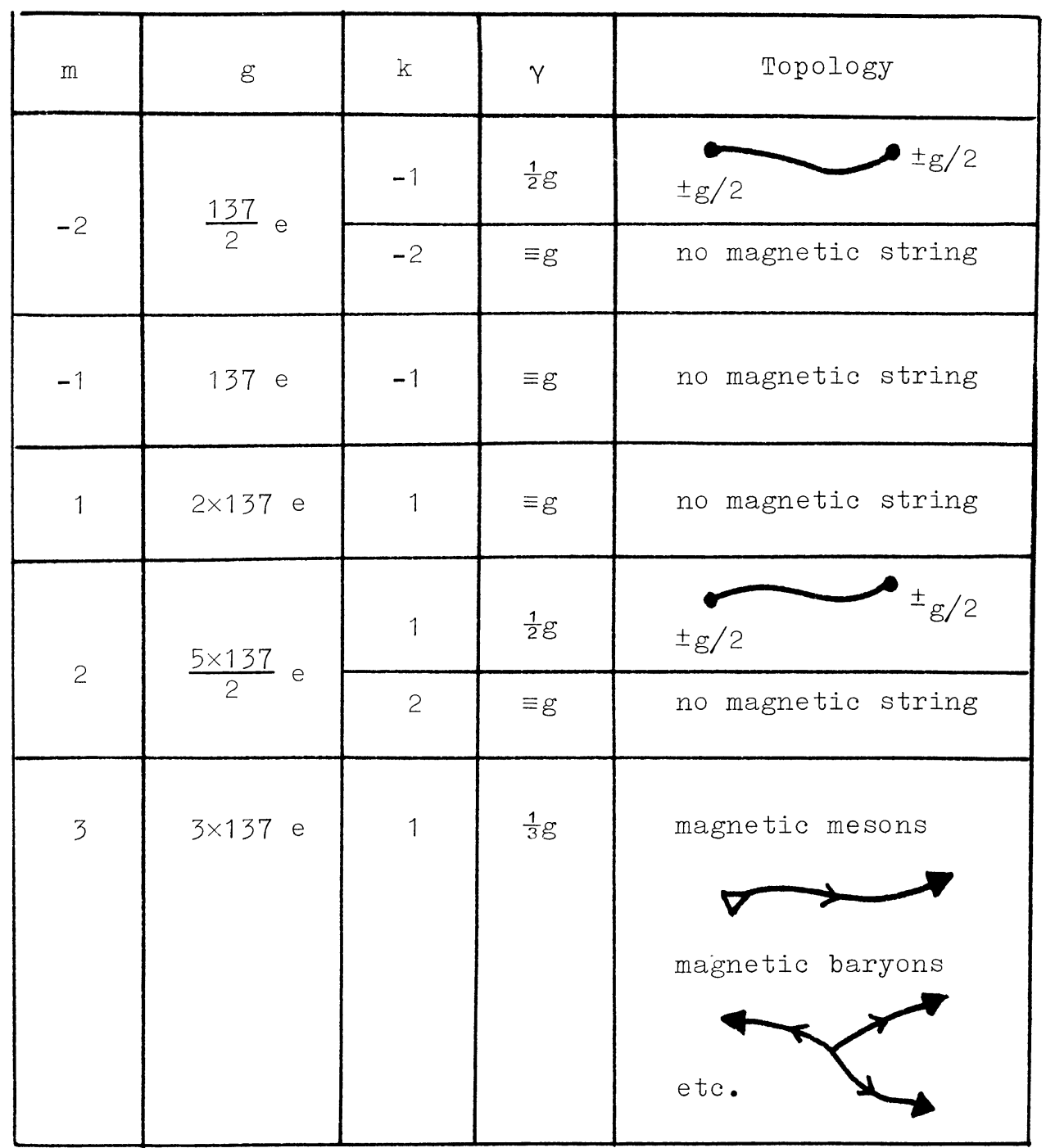

The $m=3$ solution is interesting because it is dual symmetric and the electromagnetic interaction is chiral invariant at the quantum level. The solutions with $\gamma=\frac{1}{2} g$ have strings with a more simple topology (only "mesons").

The dynamics of the string

We have put in the action the parameter $x$, which is a way of taking into account the physical effect of the string (e.g., the Regge slope, the linearly rising quark-quark potential). This is in coniradiction with our hypothesis that the "observability" of the string is due to magnetic charges. We have to consider this $x$ as a renormalized rest tension (or linear mass density) due to virtual monopole-antimonopole pairs around the string (Fig. 8), and take the "bare" $x$ to be zero. 
The action for such a history is precisely D. If we consider it as a "vacuum polarization" diagram, the corresponding $S$ matrix is $e^{i D}$ which is 1 for the electron, and $e^{ \pm 2 i \pi / 3}$ for the quarks. $x$ should then be a periodic function of the charge at the end :

$$
\begin{aligned}
& x( \pm e / 3)=x( \pm 2 e / 3)=x( \pm 4 e / 3)=\ldots \\
& 0=x( \pm e)=x( \pm 2 e)=\ldots
\end{aligned}
$$

and the mass scale would be the (bare ?) mass of the monopole. Conversely, the magnetic strings would acquire a non-zero rest tension from virtual electron-positron and quark-antiquark pairs. Their $x$ would be different.

\section{6. - CONCLUSION}

We have reviewed the significance of Dirac quantization of electric charges, and allowed it to be violated at the quark level, in order to explain the string behaviour of the hadrons. We generalized the Dirac procedure to define the classical action of a system of dyons connected by a network of strings, but introduced an extra contact interaction when two strings cross each other, which is of the order of $\hbar$. A particular value of this term restores the dual symmetry between electric and magnetic charge at the quantum level, but we considered other possibilities. The generalized Dirac condition for string 1 to be unobservable is

$$
D\left(e_{1}, g_{1} ; e_{2}, g_{2}\right)=(1-x) e_{2} g_{1}-x e_{1} g_{2} \equiv 0
$$

for all $\left(e_{2}, g_{2}\right)$, where $x$ is related to the contact interaction.

Thus, we had two kinds of strings :

- those which satisfy the (generalized) Dirac condition and are unobservable ;

- those which do not, and acquire a physical reality. The usual hadrons are made of such strings。 
We then investigated the different models of magnetic charges and magnetic strings which could explain :

- the quantization of electric charge (in units of $e / 3$ ),

- the triality and the (assumed) string structure of the hadron.

We found an infinity of solutions, some of these having no magnetic strings, some having non-oriented strings and only "mesons", some having the same topology as the usual hadrons (mesons, baryons, exotics,...). The dual symmetric solution is of this type, and in this case the smallest free magnetic charge would be $g=3 \times 137 \mathrm{e}$.

So we made a "finalist" approach to the problem of the existence of magnetic charges. It relies on the assumption that hadrons are strings with fractionally charged quarks at the ends, which is not yet proved or disproved. On the experimental side, the hunt of magnetic monopoles has been unsuccessful so far, but the huge value of the magnetic charges is itself a handicap for their separation.

One should also consider the actual development of unified theories of weak and electromagnetic interactions, and of non-Abelian theories of monopoles. Perhaps these different interactions can co-exist.

\section{ACKNOWLEDGEMENTS}

I am indebted to S. Deser and B. Zumino for enlightening discussions and I thank P. Goddard and D. Olive for comments on the manuscript. 


\section{REFERENCES AND FOOTNOTES}

1) PoA.M. Dirac - Proc.Roy.Soc。A133, 60 (1931).

2) P.A.M. Dirac - Phys.Rev. 74, 817 (1948).

3) I.I. Schiff - Phys.Rev. 160, 1257 (1967).

4) See, for instance :

J。Scherk - Revs.Modern Phys. 47, 123 (1975).

5) X. Artru and G. Mennessier - Nuclear Phys. B70, 93 (1974).

6) X. Artru - Nuclear Phys. B85, 442 (1975).

7) Our metric is $d s^{2}=d x_{\mu} d x^{\mu}=\left(d x_{0}\right)^{2}-(d \vec{x})^{2} \cdot \epsilon_{\mu \nu \rho \sigma}$ is the completely antisymmetric tensor with $\epsilon_{0123}=+1$. We work in Minkowski space for simplicity, but all what follows can be generalized to the case of a non-flat space time.

8) Our $\varphi$ corresponds to $-4 \pi G^{\dagger}$ of Ref. 2), Eq. (13)。

9) S. Mandelstam - Ann。Phys。(N.Y.) 19, 1 (1962).

10) R.P. Feynman and A.R. Hibbs - "Quantum Mechanics and Path Integrals" McGraw-Hill (1965).

11) D. Zwanziger - Phys。Rev. 176, 1489 (1968); D3, 880 (1971). 


\section{FIGURE CAPTIONS}

Figure 1 Illustration of Formula (2.14).

Figure 2 Integration contour in Eq. (2.17a).

Figure 3 Variation of the sheet swept by a magnetic string.

Figure 4 Crossing of an electric and a magnetic string.

Figure_5 The displacement $\mathrm{dX}$ of the end point and its dual vector.

Figure 6 Paths $P$ and $P^{\prime}$ when string 1 is at rest and string 2 is moving at the crossing point。

Figure 7 Paths $P$ and $P^{\prime}$ when string 2 is at rest and string 1 is moving at the crossing point.

Figure 8 "Vacuum polarization" around a string by a virtual monopoleanti-monopole pair 


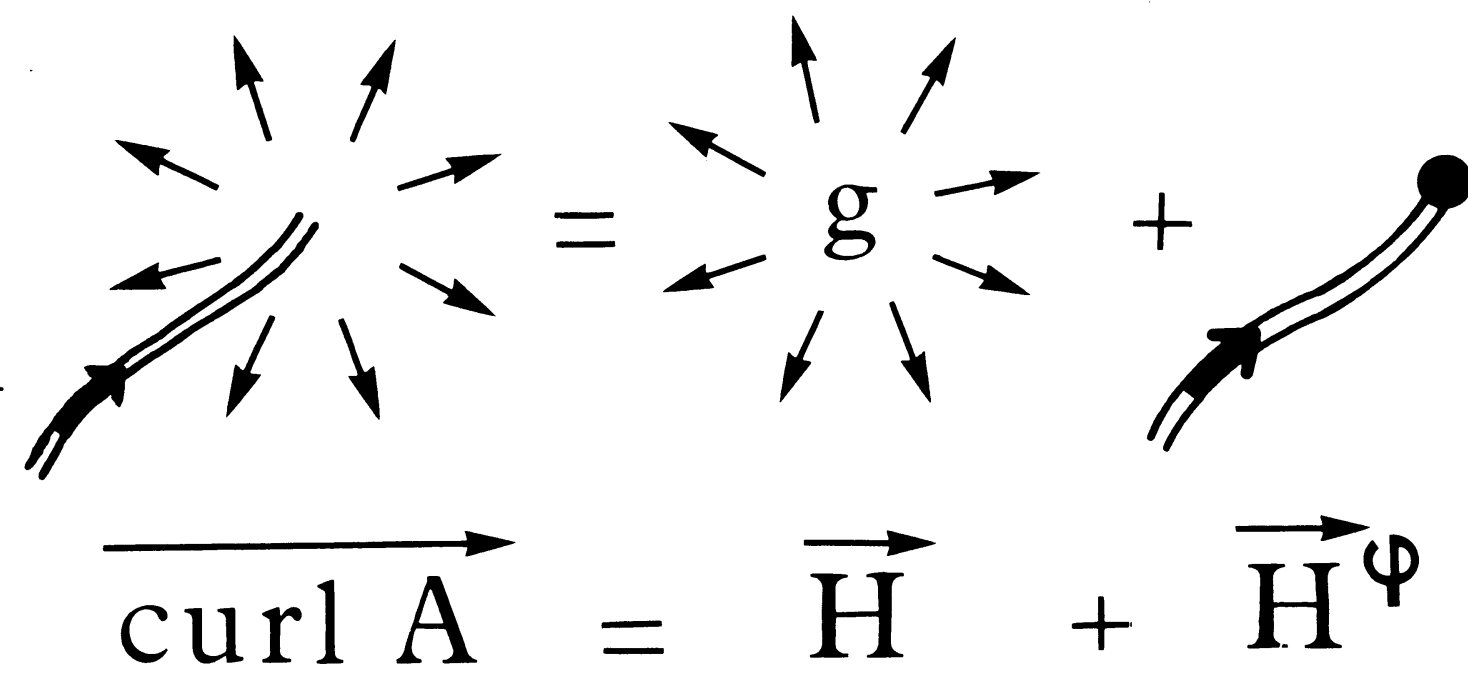

FIG. 1

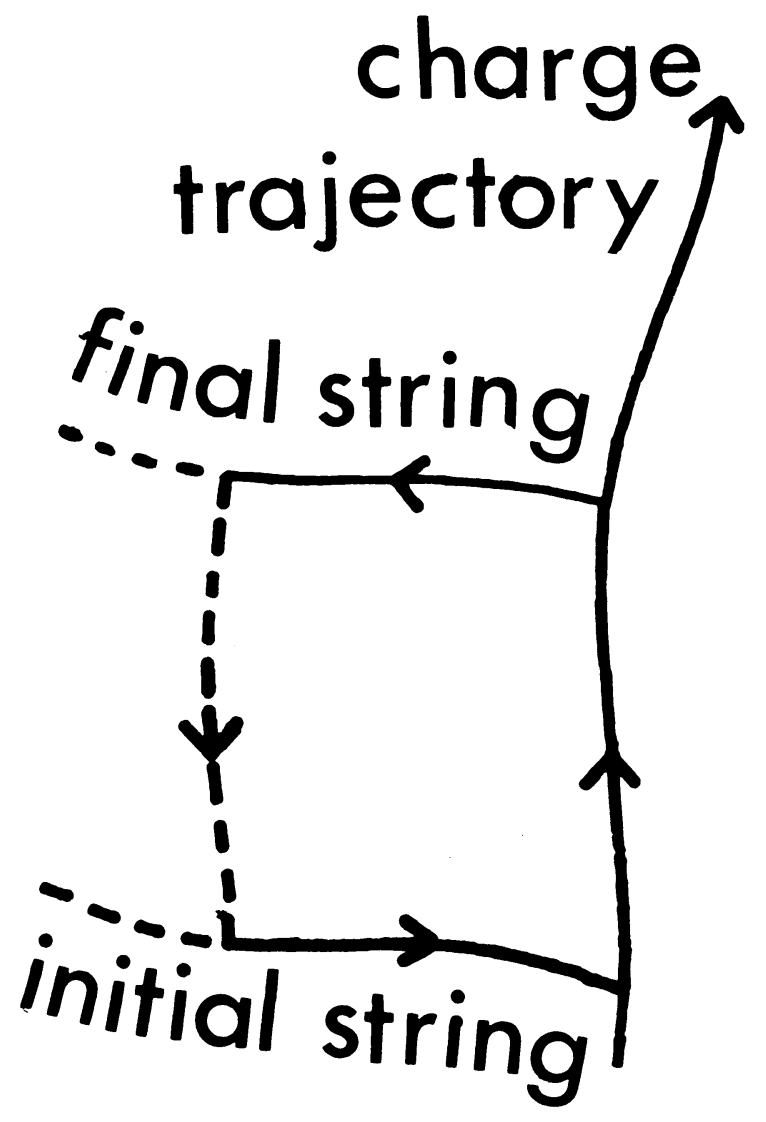

FIG. 2

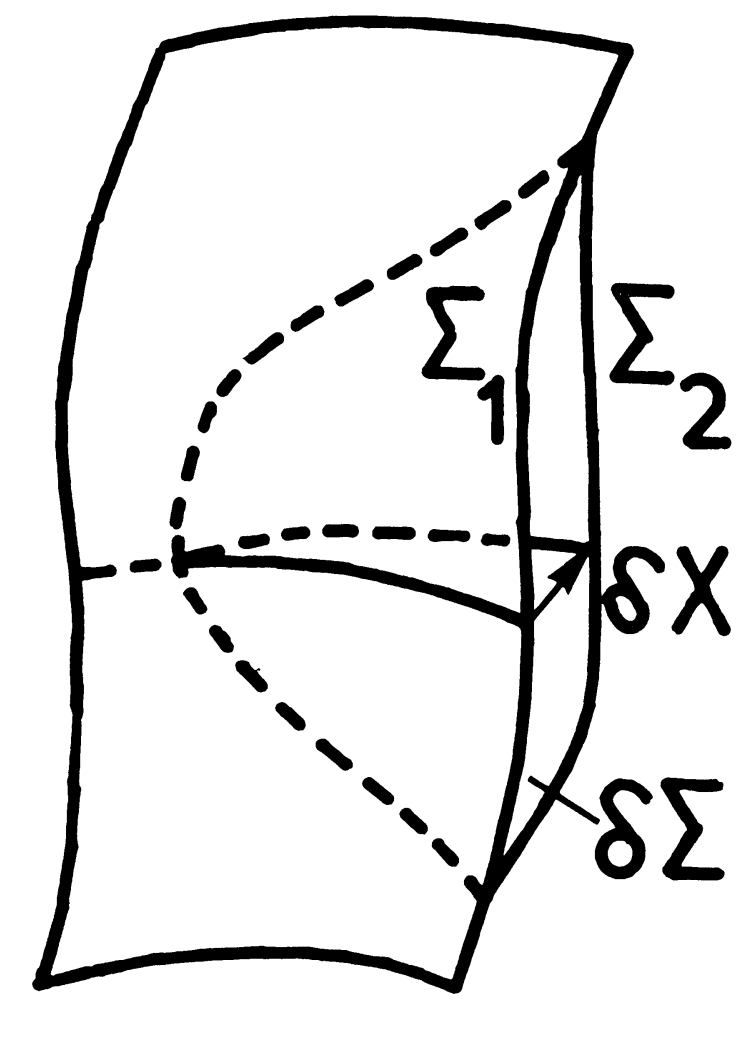

FIG. 3 

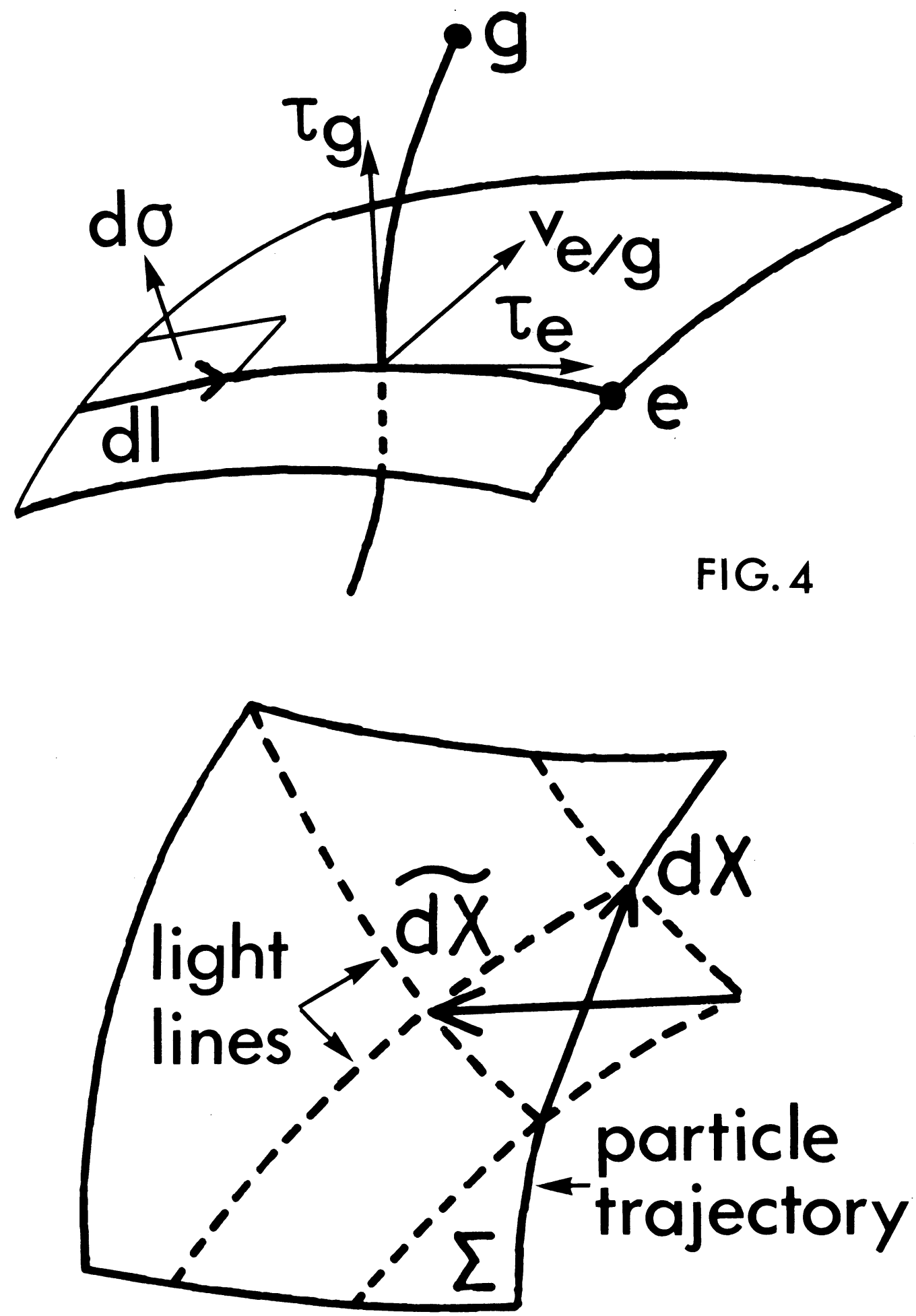

FIG. 5 


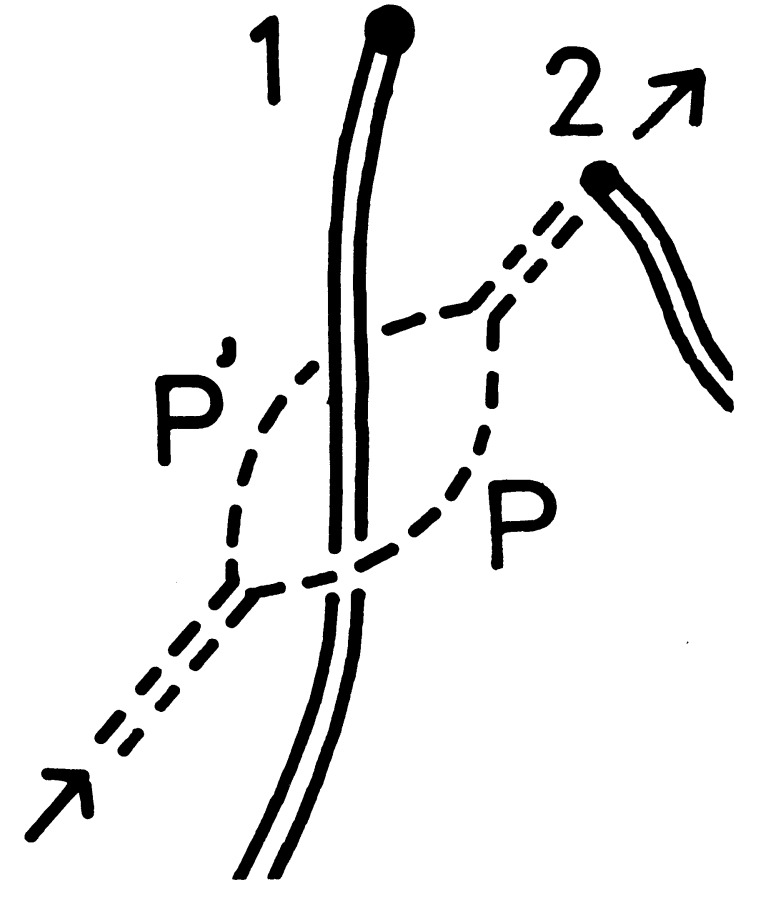

FIG. 6

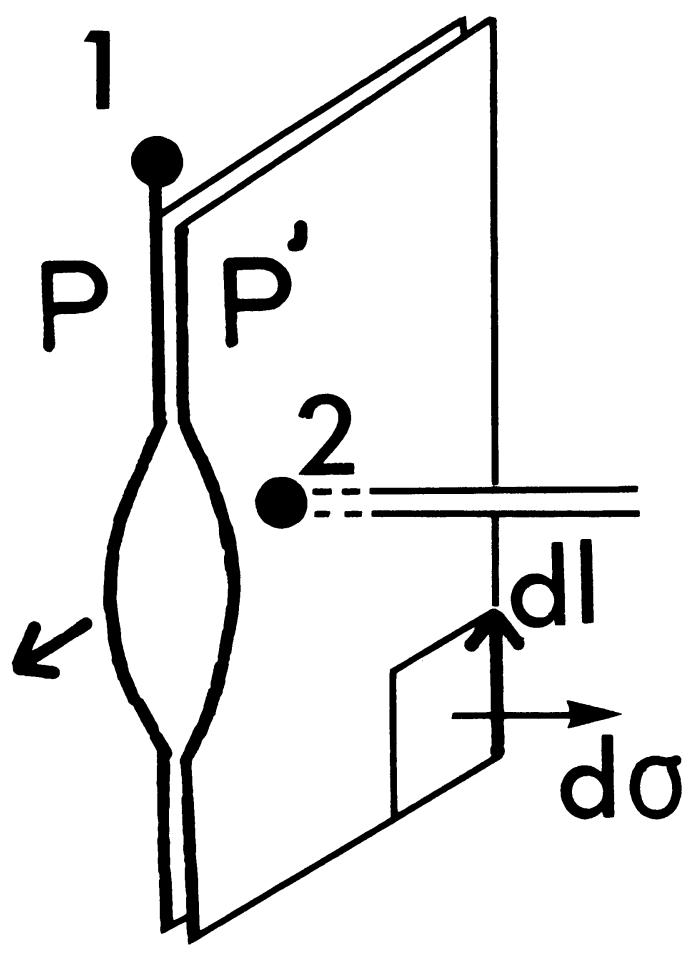

FIG. 7

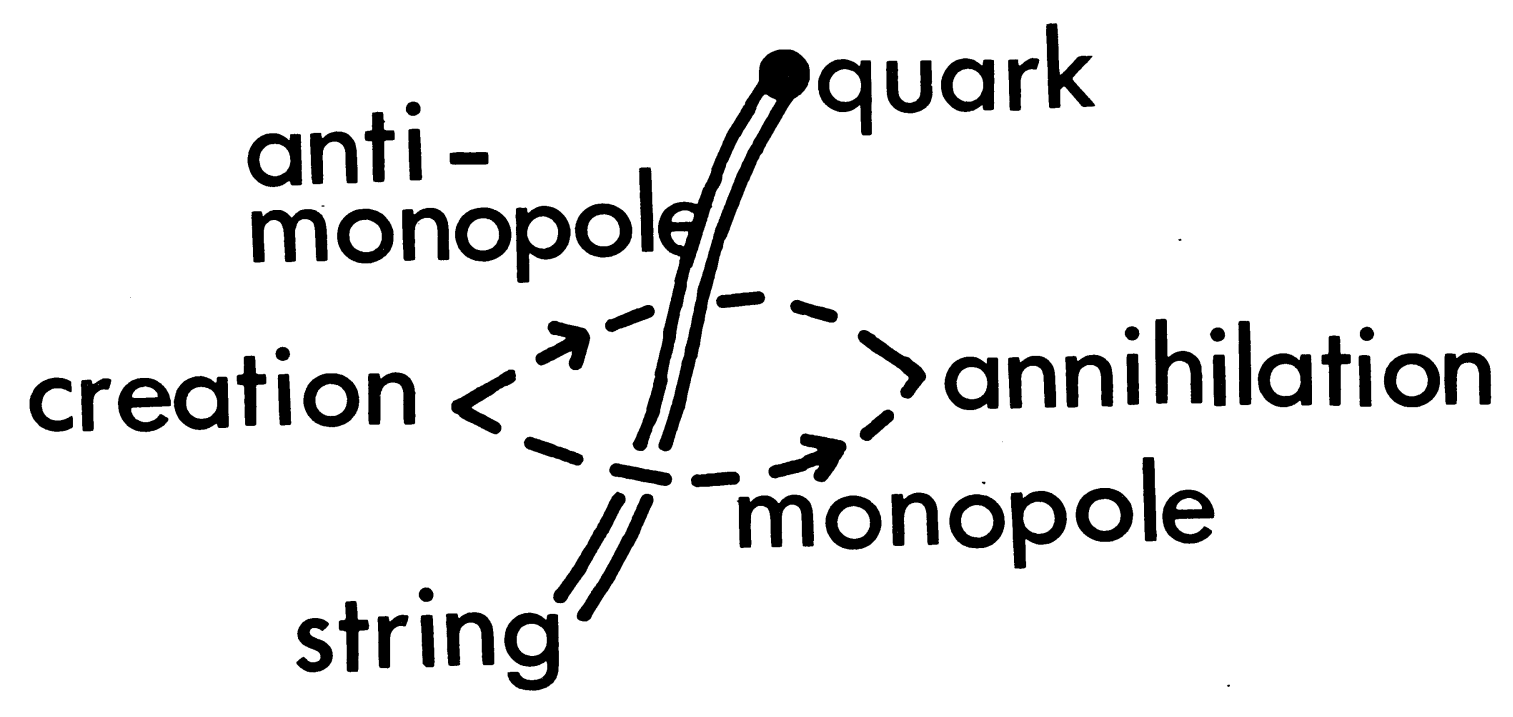

FIG. 8 